\title{
The combination of systemic therapy and
} locoregional radiotherapy prolongs survival in newly diagnosed metastatic nasopharyngeal \section{carcinoma patients}

This article was published in the following Dove Press journal:

OncoTargets and Therapy

Number of times this article has been viewed

\section{Zhenzhen Yin \\ Ximei Zhang \\ Youyou Wang \\ Peiguo Wang \\ Zhiyong Yuan}

Department of Radiation Oncology, Tianjin Medical University Cancer Institute and Hospital, Key Laboratory of Cancer Prevention and Therapy, National Clinical Research Center for Cancer, Tianjin's Clinical Research Center for Cancer, Tianjin, People's Republic of China
Correspondence: Zhiyong Yuan;

Peiguo Wang

Department of Radiation Oncology,

Tianjin Medical University Cancer Institute and Hospital, Key Laboratory of

Cancer Prevention and Therapy, National Clinical Research Center for Cancer,

Tianjin's Clinical Research Center for

Cancer, Tianjin 300060, People's Republic of China

Tel +8622 23345609

Fax +86 2223345607

Email zhiyong0524@I63.com; wpg2006@126.com
Background: The purpose of this study is to assess the role of locoregional radiotherapy (RT) when used in combination with systemic chemotherapy, for patients with newly diagnosed metastatic nasopharyngeal carcinoma (NPC), in a non-endemic region of northern China.

Methods: In total, 611 patients with NPCs were newly diagnosed between June 2011 and June 2016 following visits to our hospital; of these, 32 patients presented with metastasis at initial diagnosis. Among these 32 patients, 29 had single-organ metastasis and 3 had multipleorgan metastasis. All patients were treated with RT for local and regional disease.

Results: The median follow-up for all patients was 20 months (range 9-59 months), and median survival was not achieved (some patients had succumbed) at the time of the last follow-up. The 2-year overall survival (OS) rate was $75.2 \%$, and 3-year OS rate was $50.1 \%$. There was a significant difference between patients with single- and multiple-organ metastasis: 2-year OS was $67.5 \%$ for single- vs $0 \%$ for multiple-organ metastasis $(p=0.039)$. Patients treated with intensity-modulated RT had a better prognosis than patients treated by conventional RT: 2-year OS was $76.6 \%$ for single- vs $44.4 \%$ for multiple-organ metastasis (no significant difference was found between the 2 groups, $p=0.297$ ). For patients with progression (all were with distant disease progression), the median progression time was 8 months (6-22 months), and the median survival after disease progression was 6 months (2-14 months).

Conclusion: For patients with newly diagnosed metastatic NPCs, especially with single-organ metastasis, the addition of RT to systemic chemotherapy improved survival and disease control compared with historical cohort.

Keywords: nasopharyngeal carcinoma, metastatic, radiotherapy, chemotherapy, advanced disease

\section{Introduction}

Nasopharyngeal carcinoma (NPC), compared with other cancer types, is uncommon. With a unique pattern of geographical distribution, NPC has a significantly higher incidence in southern China than northern China. Despite being of a similar cell or tissue lineage, NPCs present with a higher propensity to metastasize to distant sites and are distinctly different from other head and neck squamous cell carcinomas. ${ }^{1}$ Most NPCs are categorized as a localized disease, with only about $5 \%-6 \%$ of patients presenting with distant metastasis at first diagnosis. ${ }^{2}$ Although significant progress has been achieved in long-term disease control for patients with early-stage and locoregional advanced disease, ${ }^{3}$ metastatic NPCs are still considered incurable. 
The absence of prospective trial data or generally accepted management guidelines has led to there being no consensus among oncologists regarding the optimal treatment for patients with newly diagnosed metastatic NPCs. To date, the mainstream approach for these patients is systemic chemotherapy with palliative intent. Although NPCs are a chemosensitive disease, with objective response rates as high as $80 \%$, the median survival after chemotherapy alone for disseminated NPCs is merely 10-15 months and the median progression-free survival (PFS) is about 6 months. ${ }^{4,5}$ Only $3 \%-8 \%$ of patients obtain complete remission after systemic chemotherapy, so most of them remain carrying primary residual disease. ${ }^{6}$ Residual local disease usually becomes a source of progression after chemotherapy is stopped, with a serious impact on patients' quality of life and survival.

Recently, there is evidence to demonstrate that there is improved overall survival (OS) for patients with extensive small-cell-lung cancer, another chemo- and radiosensitive disease, when treated by both intrathoracic radiotherapy (RT) and chemotherapy, compared to chemotherapy alone. ${ }^{7}$ As for NPCs, RT has achieved superb locoregional control in nonmetastatic disease, while its role in the metastatic setting remains a subject of debate. ${ }^{3,8}$ Therefore, with the intention of evaluating the efficiency of the addition of locoregional RT to chemotherapy at the point of initial diagnosis of metastatic NPCs, we conducted a retrospective, therapeutic study of a uniform group of newly diagnosed metastatic NPC patients in a non-endemic region of northern China. Patients were treated with systemic chemotherapy followed by definitive RT for the local and regional disease, with or without active local treatment of metastatic sites.

\section{Methods}

\section{Patient selection}

Between June 2011 and June 2016, a total of 611 NPC patients visited our hospital, of whom 32 consecutive patients presenting with distant metastasis at initial diagnosis were included in this analysis. Inclusion criteria were as follows: (1) having pathologically confirmed NPC; (2) presenting with distant metastasis at initial diagnosis based on physical examination and radiological imaging, such as computed tomography (CT), magnetic resonance imaging (MRI), emission computed tomography (ECT), or positron emission tomography (PET)/CT; (3) receiving systemic chemotherapy and RT in the nasopharyngeal and neck region; and (4) complete follow-up and clinical data are available. The Ethical Review Committee of the Tianjin Medical University Cancer Institute and Hospital approved the project. Written informed consent for their medical data to be used for research was obtained from patients and stored in the hospital database. Written informed consents of patients aged under 18 years were obtained from their parents or legal guardians.

\section{Pretreatment evaluation}

All patients received pretreatment evaluation consisting of a complete medical history, physical examination, complete blood counts, blood chemistries, urinalysis, electrocardiogram, flexible naso-endoscopic examination with biopsy, CT scans of the nasopharynx and neck, technetium-99m bone scans, ultrasound of the liver, chest X-ray, PET/CT, and dental evaluation. Tumors were restaged according to the 2010 American Joint Cancer Committee staging classification.

\section{Treatment}

Of 32 patients, 29 were treated with systemic chemotherapy. The chemotherapy regimen consisted of intravenous cisplatin (100 mg/m² delivered in 3 daily doses) and 5-fluoruouracil $\left(1,000 \mathrm{mg} / \mathrm{m}^{2}\right.$ delivered in 5 daily doses) administered every 21 days for each cycle. Induction, concurrent, and adjuvant chemotherapy were delivered in 25, 14, and 12 patients, individually. All the patients were treated with local and regional RT, and 25 patients with RT dose higher than $66 \mathrm{~Gy}$. Patients with metastatic foci in the bones, lung, liver, and non-regional lymph nodes were recommended RT, surgery, or percutaneous alcohol injection.

\section{Statistical analysis}

Statistical analysis was performed using the SPSS 19.0 package. OS and PFS were estimated by the Kaplan-Meier method and were compared using the log-rank test. A 2-tailed $p$-value less than 0.05 was considered statistically significant. OS was calculated from diagnosis until death or until the date of last follow-up. PFS was calculated from diagnosis until any disease progression or until death.

\section{Results}

\section{Patients' characteristics}

Between June 2011 and June 2016, a total of 611 patients with newly biopsy-proven NPCs visited our hospital, of whom 32 presented with distant metastasis at initial diagnosis. The median age was 48 years (17-80 years); 25 patients were men, and 7 were women. Of the 32 patients with metastatic diseases, 29 (91\%) had single-organ metastasis while 3 had multiple-organ metastases: metastatic sites were the bones, lung, liver, and distant lymph nodes. Patient characteristics are shown in Table 1. 
Table I Patients' characteristics

\begin{tabular}{ll}
\hline Patients' characteristics & $\mathbf{n}(\%)$ \\
\hline Median age, (years) & 48 years (I7-80 years) \\
Gender & $25(78 \%)$ \\
Male & $7(22 \%)$ \\
Female & \\
T stage & $3(9 \%)$ \\
T1 & $14(44 \%)$ \\
T2 & $11(34 \%)$ \\
T3 & $4(13 \%)$ \\
T4 & \\
N stage & $1(3 \%)$ \\
N0 & $6(19 \%)$ \\
NI & $20(62 \%)$ \\
N2 & $5(16 \%)$ \\
N3 & \\
Metastasis organ & $29(91 \%)$ \\
Single & $3(9 \%)$ \\
Multiple & \\
Metastasis site & $18(56 \%)$ \\
Bones & $9(28 \%)$ \\
Lung & $6(18 \%)$ \\
Liver & $2(6 \%)$ \\
Distant lymph regions &
\end{tabular}

\section{Treatment data}

Of the 32 patients studied, 29 received systemic chemotherapy; 3 patients did not receive systemic chemotherapy because 2 were older than 70 years and 1 had liver dysfunction. The median number of chemotherapy cycles was 5 (2-12). The median radiation dose to the primary disease location was $70 \mathrm{~Gy}$ (50-70 Gy), and 7 patients were treated with an RT dose lower than $66 \mathrm{~Gy}$. Ten patients were treated with 2-dimensional (2D) RT, and 22 were treated with intensity-modulated radiotherapy (IMRT). Patients with metastatic foci in the bones, lung, and non-cervical lymph nodes, who were amenable to local therapy, were treated in accordance with their physicians' decisions; treatment data are shown in Table 2.

\section{Treatment outcomes}

The median follow-up time was 20 months (range 9-59 months) for the entire group of patients. At the time of last follow-up, the median survival was not achieved. The 2 - and 3 -year OS were $75.2 \%$ and $50.1 \%$, respectively, as shown in Figure 1. Of the patients studied, 12 had distant metastatic disease progression, but none developed local failure. The median progression time was 8 months (6-22 months), and the median survival time after distant disease progression was 6 months (2-14 months).
Table 2 Treatment data

\begin{tabular}{ll}
\hline Characteristics & $\mathbf{n}(\%)$ \\
\hline RT dose & \\
$\quad \geq 66$ Gy & $25(78 \%)$ \\
$\quad<66$ Gy & $7(22 \%)$ \\
Treatment techniques & \\
2 D & $13(41 \%)$ \\
IMRT & $19(59 \%)$ \\
Number of cycles of chemotherapy & \\
0 & $3(9 \%)$ \\
$\leq 6$ & $22(69 \%)$ \\
$>6$ & $7(22 \%)$ \\
Neoadjuvant chemotherapy & \\
$\quad$ Yes & $25(78 \%)$ \\
No & $7(22 \%)$ \\
Concurrent chemotherapy & $14(44 \%)$ \\
Yes & $18(56 \%)$ \\
No & \\
Adjuvant chemotherapy & $12(38 \%)$ \\
Yes & $20(62 \%)$ \\
No & \\
Metastatic disease treatment & $10(31 \%)$ \\
Yes & $22(69 \%)$ \\
No &
\end{tabular}

Abbreviations: RT, radiotherapy; 2D, 2-dimensional; IMRT, intensity-modulated radiotherapy.

\section{Prognostic factors}

Potential prognostic factors for OS, including age, gender, radiation dose, chemotherapy cycles, and site of metastases, were evaluated using log-rank comparisons, as shown in Table 3. Except for the number of metastasized organs, other factors were not found to be predictors of OS. Patients with single-organ metastases showed a significantly improved survival compared to those with multiple-organ metastases (2-year OS $67.5 \%$ vs $0 \%, p=0.039$ ) (Figure 2). Regarding

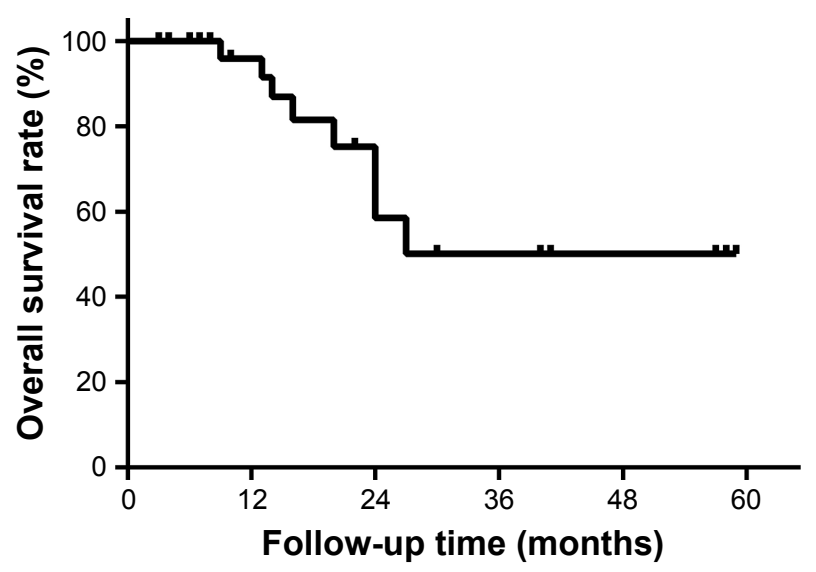

Figure I The OS curve of all patients (2-year OS was $75.2 \%$, and 3-year OS was $50.1 \%$ ).

Abbreviation: OS, overall survival. 
Table 3 Factors associated with OS

\begin{tabular}{lllll}
\hline Factors & Subgroup & 2-year OS & $\chi^{2}$ & p-value \\
\hline Age & $>48$ years & $48.5 \%$ & 0.151 & 0.698 \\
& $\leq 48$ years & $65.7 \%$ & & \\
Gender & Male & $58.9 \%$ & 0.002 & 0.967 \\
& Female & $66.7 \%$ & & \\
Number of metastatic & Single & $67.5 \%$ & 4.535 & 0.033 \\
organs & Multiple & 0 & & \\
Neoadjuvant & Yes & $74.0 \%$ & 0.101 & 0.750 \\
chemotherapy & No & $80.0 \%$ & & \\
Concurrent & Yes & $56.0 \%$ & 1.387 & 0.239 \\
chemotherapy & No & $64.3 \%$ & & \\
RT technique & IMRT & $76.6 \%$ & 1.086 & 0.297 \\
& 2D-RT & $44.4 \%$ & & \\
RT dose & 70 Gy & $70.9 \%$ & 0.028 & 0.868 \\
& $<70$ Gy & $60.0 \%$ & & \\
\hline Abbrevation & &
\end{tabular}

Abbreviations: OS, overall survival; RT, radiotherapy; IMRT, intensity-modulated radiotherapy; 2D, 2-dimensional.

chemotherapy cycles, there was no significant difference in survival between patients treated with fewer than 4 cycles of chemotherapy and those treated with more than 4 cycles. Though no significant difference in different RT techniques was noted, patients receiving IMRT showed better longterm survival than those receiving 2D-RT (2-year OS 76.6\% vs $44.4 \%, p=0.297)$. The median survival was 24 months (19-29 months) in patients treated with 2D-RT, while it was not achieved in patients treated with IMRT (Figure 3). Because only 32 patients were included in this study, Cox regression analyses were not performed.

\section{Discussion}

NPC is less common in northern China than in southern China. ${ }^{9}$ Platinum-based chemotherapy is considered the cornerstone treatment for metastatic NPCs, but was offered to only $5 \%$ of patients with NPCs at first diagnosis. ${ }^{2}$ The prognosis of NPC patients treated with palliative chemotherapy

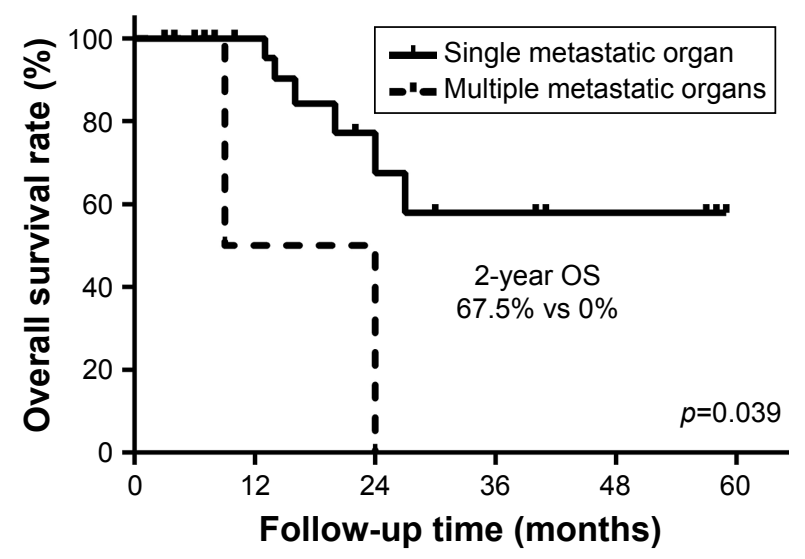

Figure 2 The OS curves of patients with single metastatic organ or multiple metastatic organs.

Abbreviation: OS, overall survival.

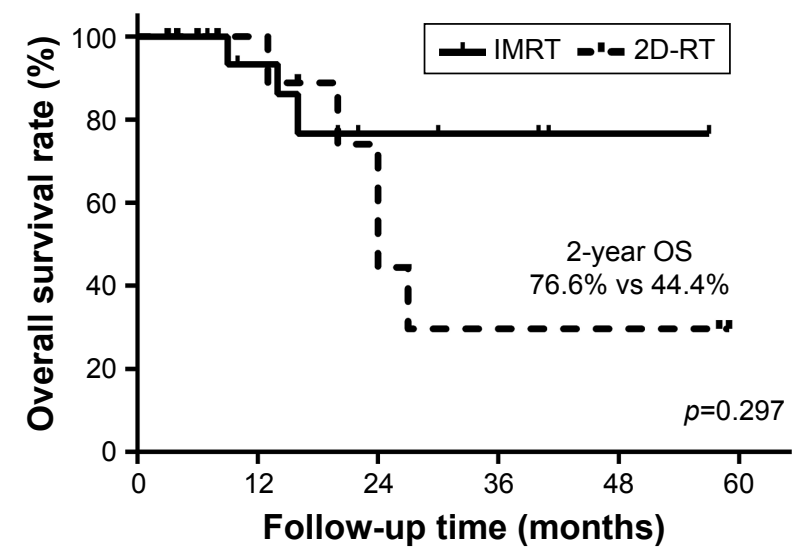

Figure 3 The OS curves of patients treated with IMRT or 2D-RT.

Abbreviations: OS, overall survival; IMRT, intensity-modulated radiotherapy; 2D-RT, 2-dimensional radiotherapy.

was quite poor, with objective response rates of $55 \%-80 \%$ and a median OS ranging from 10 to 15 months. ${ }^{4,5}$ In this retrospective study, results showed a favorable treatment outcome in a group of patients with newly metastatic NPCs in northern China treated with a combination of systemic chemotherapy and definitive doses of radiation therapy to primary disease. With a median follow-up time of 20 months, although median survival was not achieved, the 2- and 3-year OS was $75.2 \%$ and $50.1 \%$, respectively, substantially longer than the historical cohorts reported in previous studies. ${ }^{10}$ Additionally, patients with single-organ or tissue-region metastases showed better outcomes with the addition of RT, especially IMRT, than those with more extensive disease metastasized to multiple organs or tissue regions.

Newly diagnosed metastatic NPCs accounted for about $5 \%$ of NPCs in patients at their initial visit to hospital, and the treatment of this group of patients remained a great challenge. Though it is the mainstream treatment, palliative chemotherapy, albeit with high objective response rates, resulted in fewer than $10 \%$ of patients achieving a complete response for their primary disease. ${ }^{6} \mathrm{~A}$ bulky primary tumor, if left untreated, might result in severe symptoms and complications such as bleeding, visual and hearing impairment, severe headache, and cranial nerve paralyses. It should also be noted that despite the presence of metastatic disease, a subset of these patients eventually died of local nasopharyngeal failure. ${ }^{11}$ RT could significantly alleviate the burden of local disease and symptoms, and after local treatment, the primary disease recurrence was scarce, with no patient developing local failure in our results. Several retrospective studies from endemic regions showed that the combination of RT and chemotherapy seems to obtain superlative survival, with an OS ranging from 22 to 29 months, compared to chemotherapy alone. ${ }^{6,11-14}$ Thus, it is reasonable to suggest that the 
application of local and neck RT in patients with metastasis at initial diagnosis of NPC could lower local recurrence rates and prolong survival. Given the particular anatomical location of the primary site, local symptoms, and primary disease progression, it is important that the local treatment to the primary disease should aim for local disease control in patients with systemic disease. However, since all of the above-mentioned results were derived from retrospective studies, ${ }^{6,11-13}$ prospective randomized trials are warranted to further validate the efficacy and benefit of locoregional treatment. A registered Phase III randomized clinical trial that includes patients with metastatic disease treated by systemic chemotherapy in combination with or without RT for the local disease is currently in progress, and its results are eagerly looked forward to (www.ClinicalTrials.gov; NCT02111460).

Patients with a distant metastasis at initial diagnosis do not behave in a uniform manner, ${ }^{15,16}$ so deciding which group of metastatic NPCs would benefit most from locoregional RT poses a great challenge for physicians and radiation oncologists. Competing risks of locoregional or distant metastatic disease progression, and patients' tolerance must be taken into consideration. Firstly, patients with limited distant metastases may benefit more from locoregional $\mathrm{RT}$, as shown in our results, where NPC patients with single-organ metastases showed a superior survival to those with metastases to multiple organs or tissue regions. Reports also showed that the site of metastases is associated with survival, with liver metastases having poorer outcomes than lung or bone metastases. ${ }^{11}$ However, this was not the case in our results because there were only 6 patients diagnosed with liver metastases. Secondly, the response to chemotherapy is another important factor influencing patient survival. For patients with complete or partial tumor response after chemotherapy of metastatic sites, RT of the primary disease was an independently significant favorable prognostic factor (3-year OS was $59.6 \%$ vs $20.3 \%, p<0.001$, hazard ratio $[\mathrm{HR}]=0.435)$. However, for patients achieving stable disease after chemotherapy, RT to the primary disease was also associated with a longer survival (3-year OS was $24.7 \%$ vs $0 \%, p=0.003) .{ }^{11}$ In our report, RT was generally administered to patients with primary tumor who had achieved disease control of the metastatic lesions after chemotherapy. Therefore, for patients with limited distant-organ metastases and with a good response after chemotherapy (not progressive disease), the addition of locoregional RT might significantly improve outcome.

Although several studies suggested a survival advantage of locoregional RT, the timing and combination of chemotherapy in relation to RT is still undefined. There is no consensus about the number of cycles of chemotherapy, including induction, concurrent, and adjuvant chemotherapy, which may have differential effects on treatment outcomes. One study analyzed the different combinations and found that only induction-based chemotherapy was associated with significantly better survival, whereas concurrent and adjuvant chemotherapy were not. ${ }^{6}$ Increasing cycles of chemotherapy did not seem to improve patients' survival in our cohort, as no significant survival benefit was observed for the patients receiving at least 4 cycles of chemotherapy compared to those receiving fewer than 4 cycles. There is also no consensus on dose and fractions of locoregional RT for patients with advanced NPC and other head and neck cancers. ${ }^{17}$ Some studies suggested that hypofractionated RT (20 Gy/5 fractions and $50 \mathrm{~Gy} / 16$ fractions) improved symptom and disease control, though with relatively low response rates ranging from $37 \%$ to $73 \% .{ }^{18,19}$ In NPC patients, a retrospective study suggested that a definitive dose of RT (more than $65 \mathrm{~Gy}$ ) to the primary disease regions significantly improved survival, compared with a lower dose. ${ }^{12}$ Additionally, we indicate in this report that the use of IMRT resulted in better survival than conventional RT, though without a significant difference due to the small sample size. Interestingly, the 2 -year OS reported in our results (75\%) is much higher than the $50 \%$ reported by Lin et al. ${ }^{12}$ The prolonged survival in our results may be attributed mainly to the definitive RT dose delivered to locoregional disease and the usage of IMRT. Since there is a correlation between improved local control and prolonged survival, ${ }^{20}$ it is possible that the advantage of RT in locoregional control may translate to a further survival benefit for these patients. Above all, the induction-based multidisciplinary treatment with a definitive dose of IMRT is suggested as a treatment to obtain better local control and longer survival.

Various potential prognostic factors for metastatic NPC have been proposed in several retrospective studies. Performance status, advanced age, hemoglobin level, hepatic metastases, multiple metastatic sites, radiation dose to the primary tumor-bearing region, and a short metastasis-free interval were found to be independent predictors of OS in NPCs with newly diagnosed metastases. ${ }^{5,21-24}$ In our cohort, which comprised a uniform group of newly diagnosed metastatic NPC patients with good performance status, who were treated with systemic chemotherapy and high-dose radiation, we found that NPCs with single metastases, when compared to widespread metastasis, had significantly better prognosis. Long-term disease-free survival was achieved for patients with a single metastasis after this combined therapy. 
The current study demonstrates encouraging survival outcomes in patients with metastasis at initial diagnosis of NPC after using a combination of chemotherapy and locoregional RT. However, a number of limitations need to be addressed. Firstly, the sample size of our study is relatively small, with only $5 \%$ of NPCs diagnosed initially with distant metastases. Secondly, the choice of treatment modalities for the patients was at the discretion of the attending physicians, and thus, the impressive survival in our series might be affected by a possible selection bias. Thirdly, the schedule of timing and cycles of chemotherapy is heterogeneous (ie, induction, concurrent, adjuvant, or any combination) among these patients.

In conclusion, a combination of chemotherapy and RT, especially IMRT, to primary disease seems to have survival benefits for selected patients with distant metastasis at first diagnosis compared with historical cohorts. Patients with single-organ metastasis at distant sites and a good response to chemotherapy may be more suitable for locoregional RT, while prospective randomized studies are warranted to validate the benefit of the addition of locoregional RT to chemotherapy for the treatment of newly diagnosed metastatic NPCs.

\section{Acknowledgments}

The authors acknowledge the contributions of the Department of Radiation Oncology and the Department of Medical Record Library of the Tianjin Medical University Cancer Institute and Hospital for medical record provision.

\section{Disclosure}

The authors report no conflicts of interest in this work.

\section{References}

1. Lee AW, Ng WT, Chan YH, et al. The battle against nasopharyngeal cancer. Radiother Oncol. 2012;104(3):272-278.

2. Lee AW, Poon YF, Foo W, et al. Retrospective analysis of 5037 patients with nasopharyngeal carcinoma treated during 1976-1985: overall survival and patterns of failure. Int J Radiat Oncol Biol Phys. 1992; 23(2):261-270.

3. Tham IW, Hee SW, Yeo RM, et al. Treatment of nasopharyngeal carcinoma using intensity-modulated radiotherapy - the National Cancer Centre Singapore experience. Int J Radiat Oncol Biol Phys. 2009; 75(5):1481-1486

4. Loong HH, Ma BB, Chan AT. Update on the management and therapeutic monitoring of advanced nasopharyngeal cancer. Hematol Oncol Clin North Am. 2008;22(6):1267-1278.

5. Toh CK, Heng D, Ong YK, et al. Validation of a new prognostic index score for disseminated nasopharyngeal carcinoma. Br J Cancer. 2005;92(8):1382-1387.
6. Chen MY, Jiang R, Guo L, et al. Locoregional radiotherapy in patients with distant metastases of nasopharyngeal carcinoma at diagnosis. Chin J Cancer. 2013;32(11):604-613.

7. Slotman BJ, van Tinteren H, Praag JO, et al. Use of thoracic radiotherapy for extensive stage small-cell lung cancer: a phase 3 randomised controlled trial. Lancet. 2015;385(9962):36-42.

8. Lee N, Harris J, Garden AS, et al. Intensity-modulated radiation therapy with or without chemotherapy for nasopharyngeal carcinoma: radiation therapy oncology group phase II trial 0225. J Clin Oncol. 2009; 27(22):3684-3690.

9. Torre LA, Bray F, Siegel RL, et al. Global cancer statistics, 2012. CA Cancer J Clin. 2015;65(2):87-108.

10. Lee V, Kwong D, Leung TW, et al. Palliative systemic therapy for recurrent or metastatic nasopharyngeal carcinoma - how far have we achieved? Crit Rev Oncol Hematol. 2017;114(6):13-23.

11. Zeng L, Tian YM, Huang Y, et al. Retrospective analysis of 234 nasopharyngeal carcinoma patients with distant metastasis at initial diagnosis: therapeutic approaches and prognostic factors. PLoS One. 2014;9(9):e108070.

12. Lin S, Tham IW, Pan J, et al. Combined high-dose radiation therapy and systemic chemotherapy improves survival in patients with newly diagnosed metastatic nasopharyngeal cancer. Am J Clin Oncol. 2012;35(5): 474-479.

13. Hu SX, He XH, Dong M, et al. Systemic chemotherapy followed by locoregional definitive intensity-modulated radiation therapy yields prolonged survival in nasopharyngeal carcinoma patients with distant metastasis at initial diagnosis. Med Oncol. 2015;32(9):224.

14. Rusthoven CG, Lanning RM, Jones BL, et al. Metastatic nasopharyngeal carcinoma: patterns of care and survival for patients receiving chemotherapy with and without local radiotherapy. Radiother Oncol. 2017;124(1):139-146.

15. Fandi A, Bachouchi M, Azli N, et al. Long-term disease-free survivors in metastatic undifferentiated carcinoma of nasopharyngeal type. J Clin Oncol. 2000;18(6):1324-1330.

16. Setton J, Wolden S, Caria N, et al. Definitive treatment of metastatic nasopharyngeal carcinoma: report of 5 cases with review of literature. Head Neck. 2012;34(5):753-757.

17. Hodson DI, Bruera E, Eapen L, et al. The role of palliative radiotherapy in advanced head and neck cancer. Can J Oncol. 1996;6 Suppl 1:54-60.

18. Mohanti BK, Umapathy H, Bahadur S, et al. Short course palliative radiotherapy of $20 \mathrm{~Gy}$ in 5 fractions for advanced and incurable head and neck cancer: AIIMS study. Radiother Oncol. 2004;71(3):275-280.

19. Al-mamgani A, Tans L, Van rooij PH, et al. Hypofractionated radiotherapy denoted as the "Christie scheme": an effective means of palliating patients with head and neck cancers not suitable for curative treatment. Acta Oncol. 2009;48(4):562-570.

20. Peng G, Wang T, Yang KY, et al. A prospective, randomized study comparing outcomes and toxicities of intensity-modulated radiotherapy vs. conventional two-dimensional radiotherapy for the treatment of nasopharyngeal carcinoma. Radiother Oncol. 2012;104(3):286-293.

21. Teo PM, Kwan WH, Lee WY, et al. Prognosticators determining survival subsequent to distant metastasis from nasopharyngeal carcinoma. Cancer. 1996;77(12):2423-2431.

22. Khanfir A, Frikha M, Ghorbel A, et al. Prognostic factors in metastatic nasopharyngeal carcinoma. Cancer Radiother. 2007;11(8):461-464.

23. Sun $\mathrm{P}, \mathrm{Chen} \mathrm{C}$, Xia $\mathrm{Y}$, et al. The ratio of $\mathrm{C}$-reactive protein/albumin is a novel inflammatory predictor of overall survival in cisplatinbased treated patients with metastatic nasopharyngeal carcinoma. Dis Markers. 2017;2017:6570808.

24. Chee J, Loh KS, Tham I, et al. Prognostic stratification of patients with metastatic nasopharyngeal carcinoma using a clinical and biochemical scoring system. J Cancer Res Clin Oncol. Epub 2017 Aug 28. 
OncoTargets and Therapy

\section{Publish your work in this journal}

OncoTargets and Therapy is an international, peer-reviewed, open access journal focusing on the pathological basis of all cancers, potential targets for therapy and treatment protocols employed to improve the management of cancer patients. The journal also focuses on the impact of management programs and new therapeutic agents and protocols on The manuscript management system is completely online and includes a very quick and fair peer-review system, which is all easy to use. Visit http://www.dovepress.com/testimonials.php to read real quotes from published authors.

Submit your manuscript here: http://www.dovepress.com/oncotargets-and-therapy-journal 